Research paper

\title{
Vitality and Implication of Natural Products from Moringa oleifera: An Eco-Friendly Approach
}

\author{
Nyla Mubeen ${ }^{1}$, Syeda Mona Hassan ${ }^{1}$, Shahzad Sharif Mughal ${ }^{1,}$, , Syed Khurram Hassan ${ }^{2}$, \\ Asif Ibrahim $^{3}$, Huma Hassan ${ }^{4}$, Maryam Mushtaq ${ }^{1}$ \\ ${ }^{1}$ Department of Chemistry, Lahore Garrison University, Lahore, Pakistan \\ ${ }^{2}$ Institute of Quality and Technology Management, University of the Punjab, Lahore, Pakistan \\ ${ }^{3}$ Department of Mathematics, Lahore Garrison University, Lahore, Pakistan \\ ${ }^{4}$ Department of Chemical Engineering, NFC Institute of Engineering and Fertilizer Research, Faisalabad, Pakistan
}

Email address:

shezi1130@gmail.com (S. S. Mughal)

${ }^{*}$ Corresponding author

\section{To cite this article:}

Nyla Mubeen, Syeda Mona Hassan, Shahzad Sharif Mughal, Syed Khurram Hassan, Asif Ibrahim, Huma Hassan, Maryam Mushtaq. Vitality and Implication of Natural Products from Moringa oleifera: An Eco-Friendly Approach. Computational Biology and Bioinformatics. Vol. 8, No. 2, 2020, pp. 72-76. doi: 10.11648/j.cbb.20200802.16

Received: June 22, 2020; Accepted: November 2, 2020; Published: November 19, 2020

\begin{abstract}
In this study we screened Moringa oleifera for bioactive secondary metabolites and biological activity. Secondary metabolites were detected by phytochemical tests, and biological activity was confirmed through anti-oxidant assays. Phytochemicals (phenolics and flavonoids) was done by using methanol, ethanol and aqueous extracts. Phytochemical analysis of Moringa oleifera extracts was performed in terms of TPCs and TFCs, revealed that aqueous ethanolic extract offered highest TPCs (46.5 mg GAE/g DW) and TFCs (79.2 mg CE/g DW). Antioxidant activity was determined by DPPH radical scavenging activity and measure of reducing power. Results revealed that aqueous ethanolic extract showed highest radical scavenging activity and also exhibited maximum reducing potential. Reults indicates that $M$. oleifera have potential antibacterial, antifungal, antimicrobial, antiseptic, anti-inflammatory, antioxidant, anti-malarial, and anti-rheumatic activities. Antioxidant activity of leaves extract of Moringa oleifera was evaluated by 1, 1-diphenyl-2-picry 1-hydrazyl (DPPH) and reducing power. These data support Moringa oleifera as having enough potential to be used safely as a potent antioxidant.
\end{abstract}

Keywords: Antioxidant, DPPH, TPCs, Moringa oleifera, Antioxidant Activity, Phenolic Contents

\section{Introduction}

The plants have been playing an important role for humans all over the world. Many medicinal plants help to cure disease and also used in healthy products in many countries. Moringa oleifera is one of the medicinal plant belongs to Moringacea family and this is the most widely used species and mostly cultivated in asian countries like in Pakistan, India, Bangladesh [1]. The Moringa oleifera plant originated initially about 5000 years ago in northern part of india where it was known as Murungai keerai and Murungai kaai. The $M$. oleifera tree had spread to most part of South America, Africa, Asia, and southern part of North America. People in India have been using it as daily food item from many last years. $M$. oleifera plant require to be generally cultivated in those areas where climatic conditions are good for its optimum growth. By this, maximum yield could be achieved from different useable parts of $M$. oleifera get maximal amount of products for the wellbeing of mankind [2]. Moringa oleifera is well known as drumstick. Moringa oleifera is known with various names in various countries like Suhanjna in Pakistan, Sahijan in India and Sajina in Bangladesh. $M$. oleifera is enzymatically active and nutrientdense plant [3]. M. oleifera contains more than 539 biochemical activities that are completely beneficial to man [4].

All parts of $M$. oleifera plant like leaves, bark, seeds and flowers possess nutritional value and an excellent source of 
natural antioxidant $[3,4]$. $M$. oleifera is a complete nutritive plant containing vitamins, protein, various minerals including iron, magnesium, calcium, various phenolics, amino acids and beta-carotene [5]. The flowers, leaves, fruit and pods of $M$. oleifera tree are used as a highly nutritive vegetable in many countries, like in, Pakistan, Philippines, India and many parts of Africa [6-8]. Leaf extracts of $M$. oleifera have been exhibited antioxidant activity both in vivo and vitro due to large quantity of flavonoids and phenolics [9, 10]. Moringa oleifera leaves also contain to be a rich source of protein, vitamin $C, \beta$-carotene, potassium and calcium and act as a potent natural antioxidant and thus improve the shelf-life of fat containing foods due to the presence of a variety of antioxidant constituents such as carotenoids, flavonoids and ascorbic acid [11, 12].

Previous phytochemical investigation on $M$. oleifera have shown that the leaves are rich in potassium, phosphorus, vitamin $\mathrm{C}$, vitamin $\mathrm{D}$, calcium, iron, amino acids and flavonoids [13]. M. oleifera plant is the tree brought from the mind of nature to hands of man. It was recognized as the botanical of the year 2007 by the national institute of health and praised another time in 2012 -2013. It is valued all over the world due to its ability to treat more than 300 diseases. Moreover, $M$. oleifera is very important in treating different diseases like influenza, rheumatism, ascites, infection, headaches, blood impurities, chest infection, fever cholera, glandular, bronchitis, swelling, respiratory disorders, cough and sore throat $[14,15]$.

$M$. oleifera oil has remarkable value in cosmetic and is used in hair care as a skin moisturizer and conditioner. Since Egyptian times it has been used in skin ointments ). Each part of $M$. oleifera tree can be used as food product in medicines [16]. It can be easily grown in severe condition. So, $M$. oleifera plant is also named as "never die" due to its incredible ability to survive in harsh environment even in drought. Moringa oleifera is a perennial tree, grows $10-12 \mathrm{~m}$ tall, and is somewhat slender with floppy branches. Its leaves are pale green in colour, fluffy with many small leaflets $0.3-0.6 \mathrm{~cm}$ in width and $1.3-2 \mathrm{~cm}$ in length. Leaves can be stored as in powder form for a long time without refrigeration $[17,18]$. The Moringa oleifera plant is inexpensive and the most credible substitute to not only providing good nutrition, but also the prevention and cure of a lot of diseases. $M$. oleifera tree could cheaply and easily be grown and cultivated in Pakistan [19]. The present study was undertaken to investigate the phytochemical composition and antioxidant properties of the leaves parts of $M$. oleifera.

\section{Material and Methods}

\subsection{Collection of Samples}

Moringa oleifera leaves were obtained from the Lahore, Pakistan and then dried in the shade. Dried leaves were ground to fine powder by means of an electric grinder. Powder (10 g) was soaked in five different solvent systems (distilled water, 100\% methanol, $80 \%$ methanol, $100 \%$ ethanol and $80 \%$ ethanol) in Erlenmeyer flasks for three days with continuous shaking. Extract was filtered and filtrate was evaporated to obtain dried extract that was stored at $4{ }^{\circ} \mathrm{C}$.

\subsection{Phytochemical Analysis}

Phytochemical Analysis was performed, TPCs were determined by following the methods reported Pave et al. by and TFCs were determined by the procedure described by Zafar et al. with slight modifications [22-24].

\subsection{Antioxidant Activity of Plant Extracts}

Free radical scavenging activity was determined by using 2 , 2-diphenyl-1-picrylhydrazyl (DPPH) radical as described by Maung et al. with slight ammendments [20]. Free radical scavenging activity was expressed as percentage inhibition and calculated by using the following formula:

DPPH scavenging effect $(\%$ inhibition $)=\left\{\left(\mathrm{A}_{0}-\mathrm{A} 1\right) / \mathrm{A}_{0}\right) \times 100$

Where $\mathrm{A}_{0}$ is the absorbance of the control reaction and $\mathrm{A} 1$ is absorbance in the presence of all the extract samples and reference. All tests were performed in triplicate [21].

\subsection{Determination of Reducing Power}

The reducing power of the plant leaves extracts was determined according to the procedure described by Hassan et al. with slight modification. Extracts having Concentration range (2.5-10.0 mg) were mixed with the buffer of sodium phosphate solution $(5.0 \mathrm{~mL}, 0.2 \mathrm{M}, \mathrm{pH} 6.6)$ and potassium ferricyanide $(5.0 \mathrm{~mL}, 1.0 \%)$ solution. The incubation of resulting mixture was done at $50^{\circ} \mathrm{C}$ for $20 \mathrm{~min}$. Then $5 \mathrm{~mL}$ of $10 \%$ trichloroacetic acid was added in the mixture and was centrifuged for $10 \mathrm{~min}$ at $980 \mathrm{~g}$ at $5^{\circ} \mathrm{C}$ in a refrigerated centrifuge (CHM-17; Kokusan Denki, Tokyo, Japan). Two layers were formed. The $5 \mathrm{~mL}$ of upper layer of the solution was poured in beaker and diluted it with $5.0 \mathrm{~mL}$ of distilled water and ferric chloride $(1.0 \mathrm{~mL}, 0.1 \%)$ solution, and absorbance was measured at $700 \mathrm{~nm}$ using spectrophotometer (U-2001, Hitachi Instruments Inc., Tokyo, Japan). Analysis was done thrice for each sample and results were averaged [21].

\section{Results}

\subsection{Phytochemical Analysis}

Phytochemical screening of Moringa oleifera leaves's extract was performed for the recognition of secondary metabolites. Phytochemical screening was performed with absolute methanol, aqueous methanol, absolute ethanol and aqueous ethanol, deionised water extracts. Phenolics and flavonoids are the secondary metabolites in the plants [8]. These are secondary metabolites and polyphenolic which are broadly present in plants having several properties such as anticancer, anti-inflammatory attributes, free radical scavenging activity, inhibition of oxidative and hydrolytic enzymes [24, 25].

Total phenolic contents and total flavonoid contents (TFC) 
of $M$. oleifera leaves were determined as gallic acid equivalent (GAE) and catechin equivalents (CE) respectively, as presented in table 1. TPCs and TFCs in different extracts of $M$. oleifera leaves were found to be significantly $(p<0.05)$ different which ranged over 28.1-46.5 (mg GAE/g DW) and 54.2-79.2 (mg CE/g DW), respectively. It was observed that maximum amount of TPCs (46.5 mg GAE/g DW) and TFCs
(79.2 $\pm 0.11 \mathrm{mg} \mathrm{CE} / \mathrm{g} \mathrm{DW}$ ) were found to be noticeably $(p<0.05)$ higher in aqueous methanolic extract. However, the lower level of total phenolic and flavonoids were observed in aqueous extract. Our results are in line agreement with the previous study of Bonoli et al. who reported that maximum phytochemicals were obtained in aqueous ethanolic extract of barley flour [26].

Table 1. Total phenolic and total flavonoid contents of $m$. oleifera leaves.

\begin{tabular}{lll}
\hline Solvent system & Total phenolic contents (mg GAE/g DW) & Total flavonoids contents (mg CE/g DW) \\
\hline $80 \%$ ethanol & $46.51 \pm 0.58^{\mathrm{a}}$ & $79.2 \pm 0.11^{\mathrm{a}}$ \\
$80 \%$ methanol & $40.20 \pm 0.34^{\mathrm{b}}$ & $65.9 \pm 0.21^{\mathrm{b}}$ \\
Absolute ethanol & $37.54 \pm 0.43^{\mathrm{bc}}$ & $63.4 \pm 0.26^{\mathrm{bc}}$ \\
Absolute methanol & $32.78 \pm 0.55^{\mathrm{c}}$ & $61.7 \pm 0.12^{\mathrm{cd}}$ \\
Deionised water & $28.16 \pm 1.08^{\mathrm{d}}$ & $54.2 \pm 0.13^{\mathrm{d}}$ \\
\hline
\end{tabular}

Values are mean \pm SD of three samples analyzed individually in triplicate at $p<0.05$. Superscripts alphabets within the column depicted significant difference among different solvent systems.

\subsection{Antioxidant Activity}

Antioxidant activity of medicinal plants was evaluated by using different in vitro assays such as DPPH radical scavenging activity and reducing power.

\subsection{Dpph Radical Scavenging Activity}

DPPH radical scavenging activity is one of the most widely used method for screening the antioxidant activity of plant extract. The antioxidant activities of plant phytochemicals occur by preventing the production of free radicals or by scavenging free radicals [27, 28]. Prevention of the chain initiation step by scavenging various reactive species is considered an important antioxidant mode of action [29]. Antioxidant activity of the natural products can be assessed by using DPPH radical scavenging assay. DPPH is not similar to other free radicals like hydroxyl radical and superoxide anion and is not influenced by different side reactions such as metal ion chelation and enzyme inhibition. It is a rapid, simple and convenient method for screening of many samples for radical scavenging potential and is independent of sample polarity [30].

In the present study free radical scavenging activity in $M$. oleifera leaves was determined to evaluate and explore new potential sources for natural antioxidants. As DPPH forms stable molecule on accepting hydrogen atom or an electron, so it can be used in investigating radical scavenging potential [31]. DPPH concentration was reduced due to scavenging potential of $M$. oleifera leaves and results of present study showed that all extracts have free radical scavenging activity. The free radical scavenging activity of extracts of $M$. oleifera leaves was evaluated by 1, 1-diphenyl-2-picryl-hydrazyl (DPPH, and absorbance was measured at $517 \mathrm{~nm}$. Among the tested extracts the aqueous ethanol extract displayed the most potent antioxidant activity $(80.15 \%)$, followed by aqueous ethanol (76.23\%) and ethanol (71.64\%). The aqueous extract showed lower $(61.24 \%)$ activity.

The results of the present findings are in agreement with the previous study of Chunli et al. found aqueous ethanolic extract to possess antioxidant potential [32]. M. oleifera leaves works as an antioxidant and the results of previous finding have supported the potent antioxidant activity of aqueous ethanolic extract of Moringa oleifera [14]. The results of the present investigation are supported by the previous report of Dutra et al. reported that ethanolic extract of roots of $M$. oleifera possess better scavenging activity [33]. Moreover, Venkatachalam et al. also reported that leaves of $D$. gangeticum possess antioxidant properties and could serve as scavengers and primary antioxidant [34].

Table 2. Dpph radical scavenging activity of moringa oleifera leaves extract.

\begin{tabular}{ll}
\hline Solvent system & DPPH $(\mathbf{g} / \mathbf{1 0 0 g} \mathbf{D W})$ \\
\hline $80 \%$ ethanol & $80.15 \pm 0.51^{\mathrm{a}}$ \\
$80 \%$ methanol & $76.23 \pm 0.18^{\mathrm{b}}$ \\
Absolute ethanol & $71.64 \pm 0.50^{\mathrm{c}}$ \\
Absolute methanol & $62.31 \pm 0.27^{\mathrm{d}}$ \\
Deionised water & $61.24 \pm 0.39^{\mathrm{e}}$ \\
\hline
\end{tabular}

Values are mean $\pm \mathrm{SD}$ of three samples analyzed individually in triplicate at $p$ $<0.05$. Superscripts alphabets within the column depicted significant difference among different solvent systems.

\subsection{Reducing Power}

The reducing capacity of a compound may serve as a significant indicator of its potential antioxidant activity. Measurement of reducing potential can also be used to measure some antioxidant properties of plant extracts. As a result of reduction by the antioxidant compounds of extract the yellowish ferric cyanide solution was transformed into bluish green ferrous cyanide complex. The intensity of colour is supposed to be directly related to the reducing power of extract and ultimately reflect antioxidant behaviour of the plant material [34]. The extract showed an increasing trend in reducing power as the concentration increases. Among the tested extracts the aqueous ethanolic extract displayed the most potent antioxidant activity at $10 \mathrm{mg} / \mathrm{ml}$ concentration. However, the lower concentration of extracts showed lower activity in different solvents, e.g., the lowest activity was shown in aqueous extract at $10 \mathrm{mg} / \mathrm{ml}$ concentration. The greater reducing power of the aqueous alcohlic extracts of the tested plant materials might be due to high phenolic and flavonoids contents [36, 37]. 
Table 3. Reducing power of moringa oleifera leaves extracts.

\begin{tabular}{|c|c|c|c|c|}
\hline \multirow{2}{*}{ Solvent System } & \multicolumn{4}{|c|}{ Concentration (mg/ml) } \\
\hline & 2.5 & 5 & 7.5 & 10 \\
\hline $80 \%$ ethanol $^{\mathrm{a}}$ & $1.165 \pm 031$ & $1.740 \pm 034$ & $2.00 \pm 0.44$ & $2.416 \pm 0.46$ \\
\hline Absolute ethanol ${ }^{\mathrm{ab}}$ & $1.145 \pm 0.13$ & $1.624 \pm 0.19$ & $1.921 \pm 0.21$ & $2.203 \pm 0.23$ \\
\hline $80 \%$ methanol $^{\mathrm{bc}}$ & $1.142 \pm 0.31$ & $1.535 \pm 0.33$ & $1.802 \pm 0.39$ & $2.101 \pm 0.41$ \\
\hline Absolute methanol $^{\mathrm{b}}$ & $1.169 \pm 0.22$ & $1.268 \pm 0.25$ & $1.872 \pm 0.29$ & $2.002 \pm 0.33$ \\
\hline Deionised water $^{c}$ & $1.220 \pm 0.23$ & $1.386 \pm 0.29$ & $1.484 \pm 0.30$ & $1.590 \pm 0.33$ \\
\hline
\end{tabular}

Values are mean $\pm \mathrm{SD}$ of three samples analyzed individually in triplicate at $p<0.05$. Superscripts alphabets within the column depicted significant difference among different solvent systems.

\section{Discussion}

Medicinal plants are considered as a rich resources of ingredients which can be used in drug development either pharmacopoeial, non- pharmacopoeial or synthetic drugs. A part from that, these plants play a critical role in the development of human cultures around the whole world. Moreover, some plants are considered as important source of nutrition and as a result of that they are recommended for their therapeutic values. Natural products obtained from different plant species possess a variety of biologically active compounds and are subsequently being tested for pharmacological activities. An ethno botanical study of Moringa oleifera has been attempted to highlight its medicinal significance. It has been tested and shown to have antibacterial, antifungal, antimicrobial, antiseptic, anti-inflammatory, antioxidant, anti-malarial, and anti-rheumatic activities. Over the past two decades, there has been a tremendous increase in the use of herbal medicine; however, there is still a significant lack of research data in this field $[1,3]$. As our lifestyle is now getting techno-savvy, we are moving away from nature. As herbs are natural products they are free from side effects, they are comparatively safe, eco-friendly and locally available. Traditionally there are a lot of herbs used for the ailments related to different seasons. There is a need to promote them to save the human lives [2]. These herbal products are today are the symbol of safety in contrast to the synthetic drugs, that are regarded as unsafe to human being and environment. Although herbs had been priced for their medicinal, flavoring and aromatic qualities for centuries, the synthetic products of the modern age surpassed their importance, for a while. However, the blind dependence on synthetics is over and people are returning to the naturals with hope of safety and security. It's time to promote them globally.

\section{Conclusion}

All important phytochemicals were found to be present in all extracts. Reults indicates that M. oleifera have potential antibacterial, antifungal, antimicrobial, antiseptic, anti-inflammatory, antioxidant, anti-malarial, and anti-rheumatic activities. Antioxidant activity of leaves extract of Moringa oleifera was evaluated by 1, 1-diphenyl-2-picry 1-hydrazyl (DPPH) and reducing power. This plant was selected due to the presence of different compounds of medicinal value and to determine the antioxidant capacities of these compounds. We have concluded that the aerial parts (leaves) of Moringa oleifera can be safely used as antioxidant and proves its importance as a potential antimicrobial drug.

\section{Conflict of Interest}

The authors declare that they have no competing interests.

\section{References}

[1] M. A. Maung, P. Rd. Antioxidant and Anticancer activities of Moringa oleifera Leaves. J Med plant Res. 8 (2014) 318-325.

[2] L. J. Fuglie. The Miracle Tree: Moringa oleifera: Natural Nutrition for the Tropics. Church World Service, Dakar; revised in 2001 and published as The Miracle Tree: The Multiple Attributes of Moringa. 68 (2001) 172.

[3] K. T. Mahmood, T. Mugal, Urooj, Moringa oleifera; a natural gift; A review, J. Pharma. Sci. Res. 2 (2009) 775-781.

[4] F. Anwar, A. Jamil, A. Iqbal, M. Sheikh, Antioxidant activity of various plant extracts under ambient and accelerated storage of sunflower oil, Grasas. Aceites. Sevilla. 57 (2006) 189-197.

[5] G. Mishra, P. Singh, R. Verma, S. Kumar, S. Srivastav, K. K. Jha, R. L. Khosa, Traditional uses, phytochemistry and pharmacological properties of Moringa oleifera plant: An overview, Der. Pharmacia. Letter. 3 (2011) 141-164.

[6] S. Debnath, D. Guha, Role of Moringa oleifera on enterochromaffin cell count and serotonin content of experimental ulcer model, Indian. J. Exp. Biol. 45 (2007) 726-731.

[7] F. Anwar, S. Latif, M. Ashraf, H. A. Gillani, Moringa Oleifera; A Food Plant with multiple Medicinal uses, Wiley. Inter. Sci. Phytother. Res. 21 (2007) 17-25.

[8] P. Siddhuraja, K. Becker, Antioxidant properties of various solvent extracts of total phenolic constituents from three different agro climatic origins of drumstick tree (Moringa oleifera lam) leaves, J. Agric. Food. Chem. 51 (2003) 2144-2155.

[9] B. Vongsak, P. Sithisarn, S. Mangmool, Maximizing total phenolics, total flavonoids contents and antioxidant activity of Moringa oleifera leaf extract by the appropriate extraction method, Ind. Crops. Prod. 44 (2013) 566-571.

[10] C. J. Dillard, J. B. German, Phytochemicals; Nutraceuticals and human health: A review. J. Sci. Food. Agric. 80 (2000) 1744-1756. 
[11] M. I. Bhanger, F. Anwar, Analytical characterization of Moringa oleifera seed oil grown in temperate regions of Pakistan, J. Agric. Food. Chem. 5 (2003) 6558-6563.

[12] W. A. Khateeb, E. Hussein, In vitro propagation and characterization of phenolic contentalong with antioxidant and antimicrobial activities of Cichorium pumilum Jacq, Plant. Cell. Tissue. Organ. Culture. 110 (2012) 103-110.

[13] F. Anwar, M. Ashraf, M. I. Bhanger, Analytical characterization of Moringa oleifera seed oil grown in temperate regions of Pakistan. J. Agric. Food. Chem. 51 (2005) 6558-6563.

[14] N. A. Eman, M. oleifera leaves possible uses as environment friendly material review, Int. J. Chem. Envir. Bio. Sci. 2 (2014) 141-145.

[15] J. D. Souza, A. R. Kulkarni, Comparative studies on nutritive values of tender foliage of seedlings and mature plants of Moringa oleifera Lam, J. Econ. Taxon. Bot. 17 (1993) 479 485 .

[16] S. A. Delouee, M. Aalam, A. Urooj, Drumstick; A potential source of natural Lipid Antioxident, J. Food. Process. Eng. 3 (2011) 947-959.

[17] M. Mbikay, Therapeutic potential of Moringa oleifera leaves in chronic hyperglycemia and dyslipidemia: A review, Front. Pharmacol. 3 (2012) 1-12.

[18] K. T. Mahmood, T. Mugal and Urooj, Moringa oleifera; a natural gift; A review, J. Pharma. Sci. Res. 2 (2009). 775-781.

[19] M. Elangovan, M. S. Dhanarjan, P. Mathi and N. Bhogireddy, Analysis of Phytochemicals, Antibacterial and Antioxident activities of Moringa Oleifera leaves. Int. J. Drug. Dev. Res. 6 (2014) 173-180.

[20] M. A. Maung and P. Rd, Antioxidant and Anticancer activities of Moringa oleifera Leaves, J. Med. Plant. Res. 8 (2014) 318-325.

[21] F. A. G Hassan and M. A. Ibrahim, M. Oleifiera is more nutritious and multipurpose tree, Int. J. Sci. Res. 3 (2013) 2250-3153.

[22] R. Paven, H. A. Ahad, G. N. Babu and P. S. Keerthi, Moringa oleifera phyto-constituents and its therapeutic Actions; A Review. Int. J. Res. Phar. Life. Sci. 4 (2016) 60-64.

[23] H. A. Zafar, J. S. Ali, A. Haq and M. Zia, Effect of ZnO Nanoparticles on Brassica nigra seedlings and stem explants: Growth dynamics and antioxidative response, Front. Plant. Sci., 7 (2016) 535.

[24] V. Dewanto, X. Wu, K. Adom, R. H. Liu, Thermal processing enhances the nutritional value of tomatoes by increasing total antioxidant activity. J. Agri. Food. Chem. 50 (2002). 3010-3014.
[25] Y. T. Wang, B. K. Shun, Bioactive flavenoids in medicinal plants; Struture, activity and biological fate. Asian J. Pharm. Sci. 13 (2018) 12-23.

[26] M. Bonoli, V. Verardo, E. Marconi, M. F. Caboni, Antioxidant phenols in barley (Hordeum vulgare L.) flour: comparative spectrophotometric study among extraction methods of free and bound phenolic acids, J. Agric. Food Chem. 52 (2004) 5195-5200.

[27] D. Amic, D. Beslo, N. Trinajstic, Structure radical scavenging activity relationships of flavonoids, Croat. Chem. Acta. 76 (2003) 55-61.

[28] G Oboh, R. L. Puntel, J. B T. Rocha, Hot pepper (Capsicum annuum, Tepin and Capsicum chinese, Habanero) prevents $\mathrm{Fe}^{2+}$ induced lipid peroxidation in Brain: in vitro, Food Chem. 102 (2007) 178-185.

[29] K. Dastmalchi, H. J. D. Dorman, M. Kosar, R. Hiltunen, Chemical composition and in vitro antioxidant evaluation of a water soluble Moldavian balm (Dracocephalum moldavica L.) extract, Food Sci. Technol. 40 (2007) 239-248.

[30] G. Hemant, R. Pathak, R. Arora, H. Gautum, Assessment of antibacterial and free radical scavenging activity in psychrophilic Arthrobacter sp, Pharmacology online. 1 (2011) 344-355.

[31] Y. R. Lu, F. L. Yeap, Antioxidant activities of polyphenols from sage (Saliva officinalis), Food chem. 75: (2001) 197-202.

[32] S. Chunli, W. Zhengshuang, W. Ziyan, Z. Hongcheng, Effect of Ethanol/Water Solvents on Phenolic Profiles and Antioxidant Properties of Beijing Propolis Extracts. Evid. Based Complement Alternat. Med. (2015) 9-16.

[33] R. C. Dutra, M. N. Leite, N. R. Barbosa, Quantification of phenolic constituents and antioxidant activity of Pterodon emarginatus vogel seeds. Inter. J. Mol. Sci. 9, (2008) 606-614.

[34] U. Venkatachalam, S. Muthukrishnan, Free radical Scavenging activity of Ethanolic extract of Desmodium gangeticum. J acute Med. 2 (2): (2012) 36-42.

[35] Y. Zou, Y. Lu, D. Wei, Antioxidant activity of a flavonoid rich extract of Hypericum perforatum L. In- vitro. J. Agric. Food Chem. 52: (2004) 5032-5039.

[36] Z. Cheng, L. Su, J. Moore, K. Zhou, M. Luther, J. J. Yin, L. L. $\mathrm{Yu}$, Effect of postharvest treatment and heat stress on availability of wheat antioxidants. J. Agric. Food Chem. 54: (2006) 5623-5629.

[37] B. Sultana, F. Anwar, R. Przybylski, Antioxidant activity of phenolic components present in barks of barks of Azadirachta indica, Terminalia arjuna, Acacia nilotica, and Eugenia jambolana Lam. Trees, Food Chem. 104: (2007) 1106-1114. 